\title{
Low temperature plasma processing of platinum porphyrins for the development of metal nanostructured layers
}

Alejandro Nicolas Filippin, Juan Ramon Sanchez-Valencia, * Jesús Idígoras, Manuel MaciasMontero, Maria Alcaire, Francisco Javier Aparicio, Juan Pedro Espinos, M. Carmen LopezSantos, Fabian Frutos, Angel Barranco, Juan Antonio Anta and Ana Borras*

AN Filippin, JR Sanchez-Valencia, M Macias-Montero, M Alcaire, FJ Aparicio, JP Espinos, MC Lopez, A Barranco, A Borras

Nanotechnology on Surfaces Laboratory, Materials Science Institute of Seville (ICMS-CSICUS). C/ Americo Vespucio 49, 41092, Seville (Spain)

J Idígoras, JA Anta

Departamento de Sistemas Físicos, Químicos y Naturales Universidad Pablo de Olavide 41013 Seville (Spain).

F Frutos

Departamento de Física Aplicada, E.T.S. Ingeniería Informática (Universidad de Sevilla), Avd. Reina Mercedes s/n, 41012 Seville (Spain)

Corresponding Authors: jrsanchez@icmse.csic.es; anaisabel.borras@icmse.csic.es

Keywords: Plasma processing, platinum porphyrin, Neutral density filter, Solar cell, Counter electrode

This article stablishes the bases for a vacuum and plasma supported methodology for the fabrication at mild temperatures of nanostructured platinum in the form of porous layers and nanocolumns using platinum octaethylporphyrin (PtOEP) as precursor. In addition, the application of these materials as tunable optical filters and nano-counterelectrodes is proved.

On one hand, the transparency in the ultraviolet-visible-near infrared (UV-Vis-NIR) range can be adjusted precisely between $70 \%$ and $1 \%$ by tuning the deposition and processing conditions, obtaining a high spectral planarity. Deviations of the spectra from an ideal flat filter are below $4 \%$, paving the way to the fabrication of neutral density filters. The transparency limit values yield a sheet resistivity of $\sim 1350 \Omega / \square$ and $120 \Omega / \square$, respectively. On the other hand, the catalytic properties of the nanostructures are further demonstrated by their implementation as counter electrodes of excitonic solar cells. surpassing the performance of commercial platinum as counter electrode in a $20 \%$ of the overall cell efficiency due to 
simultaneous enhancement of short-circuit photocurrent and open-circuit photovoltage. One of the most interesting features of the developed methodology is its straightforward application to other metal porphyrins and phthalocyanines readily sublimable under mild vacuum and temperature conditions.

\section{Introduction}

The development of new fabrication methods for the control on the nanostructure is crucial to synthesize rationally designed materials with enhanced properties. ${ }^{1,2}$ Plasma activated deposition methods including plasma enhanced chemical vapour deposition (PECVD), plasma assisted atomic layer deposition (P-ALD) and magnetron sputtering techniques have evolved during the last decade from focus on compact thin films towards the controlled deposition of nanoscale materials including nanoporous layers, low dimensional carbon materials and complex one-dimensional nanostructures. ${ }^{3-5}$ Advantages of these vacuum-plasma methods are mainly related to their straightforward scalability, low deposition temperatures, compatibility with an ample variety of substrates and high accuracy in the composition (including doping) of the deposited materials as well as on the control on their morphological characteristic as microstructure, texture or alignment. ${ }^{3-5}$ However, a critical bottleneck confining the application of procedures such as PECVD and P-ALD for the deposition of nanoscale materials is the limited availability of volatile metalorganic and metal halide precursors. ${ }^{6}$ Within this context, we present herein the development of a full vacuum and plasma supported protocol based on the use of metal-porphyrin, concretely, PtOEP molecules as precursor for the growth of porous Pt layers and nanocolumns. Importance of this methodology relays on both, quality of the nanostructured platinum layers fabricated at mild temperatures and straightforward applicability to an ample variety of vacuum sublimable and commercial available metalorganic molecules like 
metal porphyrins and phthalocyanines. So far, the extended use of metal porphyrins and phthalocyanines as precursor for the plasma deposition of the corresponding metal or metal oxide counterparts has been stopped due to the required post deposition processing including high temperature annealing. ${ }^{7}$ Thus, previous attempts to the deposition of platinum layers by plasma activated approaches have been focused on sputtering techniques and combination of sputtering with PECVD in order to form composite nanomaterials with interesting applications in electrocatalysis, as for instance as active layer in fuel cells. ${ }^{7,8}$ A reliable method for the fabrication of nanoscale platinum materials might play an important role in many other fields ranging from nanosensors to light harvesters. ${ }^{9,10}$ For instance, in this latter topic, the formation of electrodes with tuneable optical properties is critical for the development of high efficient solar cells. ${ }^{11,12}$ Concretely, platinum counter electrodes has been widely applied for the efficient regeneration of the liquid electrolyte in dye sensitized solar cells. ${ }^{13}$ Thus, the use of tailored porous conductive nanostructures appears as a promising route due to their inherent high surface area, ${ }^{11,14-16}$ improved catalytic performance, long-term chemical stability and relatively high transparency. ${ }^{17,18}$ In this article, we will approach to this issue from the point of view of the fabrication of supported porous $\mathrm{Pt}$ in the form of layers and nanocolumns, i.e., Pt nanoelectrodes in 2D and 1D architectures, respectively. Two different methodologies have been utilized in the deposition of the PtOEP, namely physical vapour deposition of small molecules (OPVD) $)^{19-21}$ and remote plasma assisted vapour deposition (RPAVD) ${ }^{22-25}$ both of them scalable up to wafer level and carried out at room temperature. In addition, these methods have been demonstrated to be compatible with an ample range of processable substrates including polymers, $\mathrm{Si}$, ITO, metal electrodes, self-assembled organic nanostructures and photonic architectures such as artificial opals. ${ }^{22-26}$ OPVD is a well stablished methodology for the fabrication of two dimensional organic and 
metal-organic layers also extended in the last years to the formation of supported small-molecule organic nanowires. ${ }^{19-21}$ RPAVD allows the formation of nanocomposite thin films where the functional molecules appear embedded in a highly cross-linked organic matrix consisting on the molecular fragments formed by interaction with the plasma species. ${ }^{22-25}$ In this article, we include the first results on the fabrication by RPAVD of nanostructured films from metal-organic small molecules including oxygen rich plasma gases. The post-processing of the PtOEP precursor layers to form the metal nanostructures is achieved by plasma etching under different combinations of oxygen, argon and hydrogen gases in the plasma and for temperatures ranging from $\mathrm{RT}$ to $180^{\circ} \mathrm{C}$. Herein we will refer to the etching treatment as "soft" plasma etching (SPE) ${ }^{27,28}$ to stress the mild conditions during the experiments in comparison with standard etching procedures since, in our case, the samples were emplaced facing down with respect to the plasma source.

The article is structured as follows. First, microstructure, structure, composition and optical and electrical properties of samples deposited and treated under different conditions are thoroughly analysed. As we will show below, the formation of platinum layers with well-defined nanostructure allows the fabrication of optical density filters with tuneable transparency. Then, as a proof of concept, the metallic Pt layers and nanocolumns are tested as nanocatalysers in the counter electrode of dye-sensitized solar cells made of nanoparticulate $\mathrm{TiO}_{2}$ photoelectrodes and compared the results to commercial available Platisol® counter electrodes.

\section{Results and discussion}

\subsection{Microstructure and Chemical composition}

In order to facilitate the exposition of results, the labels of the samples specifically address the main experimental parameters varied during their fabrication. As an 
example: OPVD $(550 \mathrm{~nm})+\operatorname{SPE}\left(\mathrm{O}_{2}+\mathrm{Ar}\right) 160^{\circ} \mathrm{C} 40 \mathrm{~min}+\operatorname{Anneal}\left(\mathrm{H}_{2}+\mathrm{Ar}\right)$; where $(550 \mathrm{~nm})$ indicates the thickness of the precursor layer measured by SEM, SPE $\left(\mathrm{O}_{2}+\mathrm{Ar}\right)$ the composition of the gas during the soft plasma etching (SPE) process, $160{ }^{\circ} \mathrm{C}-40$ min the substrate temperature and treatment duration during SPE and Anneal $\left(\mathrm{H}_{2}+\mathrm{Ar}\right)$ addresses that the sample was annealed under $\left(\mathrm{H}_{2}+\mathrm{Ar}\right)$ at $135^{\circ} \mathrm{C}$ as final reduction step. Since the post-annealing treatments were every time carried out under the same conditions, the label not always includes this step for the sake of simplicity and it is only indicated when comparing samples previous and after post-annealing. The plasma assisted deposition methodology (and plasma post-processing) developed in this work provides a new route to control the microstructure of the resulting thin films. Figures 1 and S1 to S3 in the Supporting Information (SI) summarize the ample microstructural variety of PtOEP and Pt containing thin layers obtained. Figure S4 gathers information of the surface topography and roughness of some characteristic samples obtained by Atomic Force Microscopy (AFM). Samples fabricated by OPVD conditions (Fig. 1 a) grow as stacked columns of inhomogeneous shapes and thicknesses that increased in length as a function of the deposition time. Samples deposited by RPAVD-Ar presented a homogeneous and continuous cross section with a smooth surface (Fig. 1 b), which can be attributed to $\mathrm{Ar}^{+}$bombardment and a partial plasma polymerization of the metal-organic precursor. ${ }^{23,24,29}$ Meanwhile, samples deposited under oxygen plasma, i.e. RPAVD-O $\mathrm{O}_{2}$, developed a 1D microstructure characterized by the homogeneous formation of vertical columns of constant diameter $(<100 \mathrm{~nm})$. The normal view SEM images of these samples (see Fig. S3a) show that the nanocolumns form agglomerates at the surface. It is worth to mention that the microstructure of the OPVD and RPAVD layers was similar independently of the type of substrate utilized ( $\mathrm{Si}(100)$ wafers, fused silica and commercial available FTO or ITO thin films on glass), although for SEM characterization a conductive substrate is 
mandatory due to the insulating nature of the samples. Even on a Si conductive

substrate, the samples cannot be perfectly focused on the microscope as it is apparent on the cross sectional images on Figure 1 a-c).

The microstructure and surface roughness of the samples were drastically affected by all the SPE treatments carried out. Two types of SPE treatments were carried out attending to the plasma gas composition, $\left(\mathrm{O}_{2}+\mathrm{Ar}\right)$ and $\left(\mathrm{O}_{2}+\mathrm{H}_{2}\right)$. Substrates temperatures and treatment durations were settled as detailed in Experimental Section. The final resulting microstructure is influenced by the fabrication process, initial thickness, the SPE treatment and its duration.

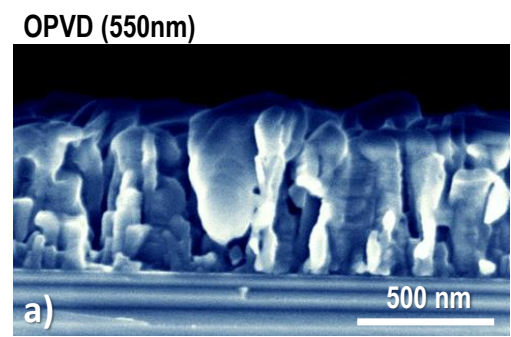

RPAVD-Ar (140nm)

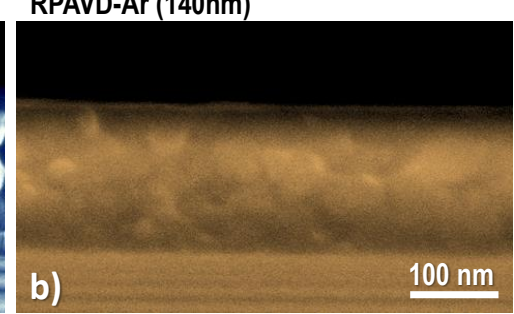

OPVD $(190 \mathrm{~nm})+\operatorname{SPE}\left(\mathrm{O}_{2}+\mathrm{Ar}\right) 160^{\circ} \mathrm{C} 40 \mathrm{~min}$
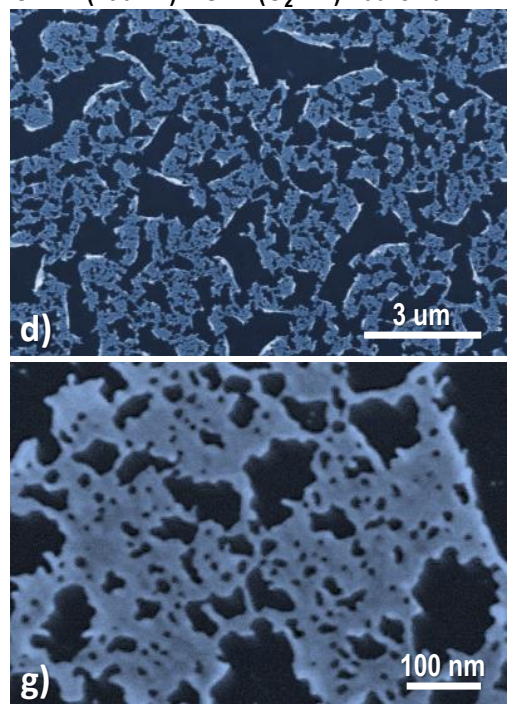
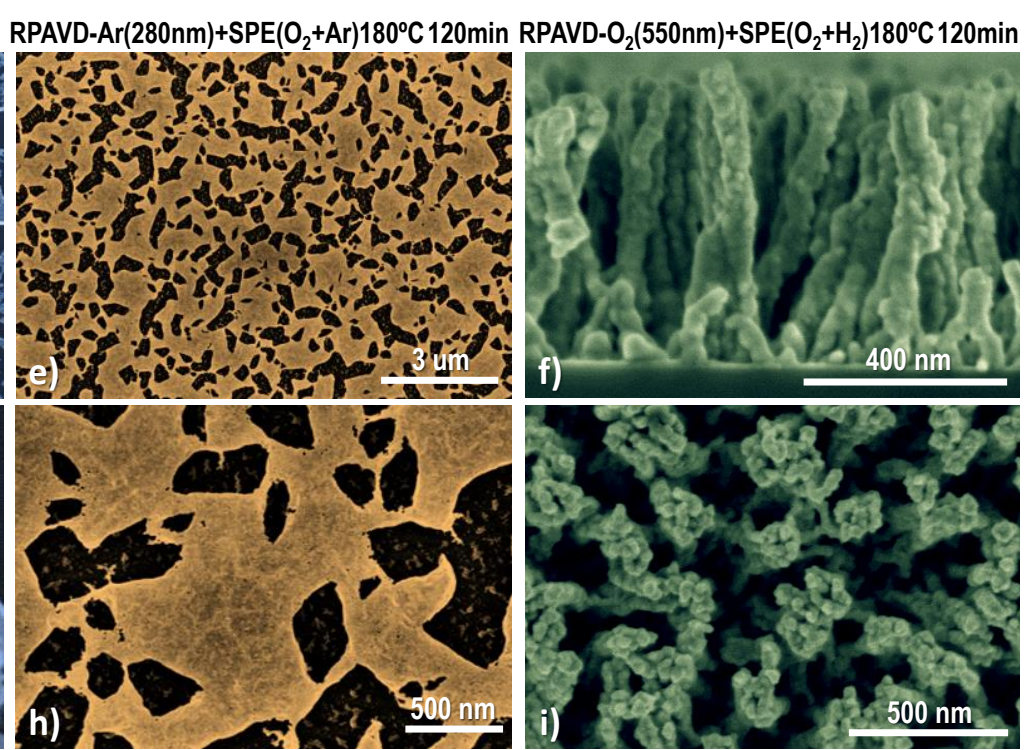

RPAVD-O 2 (550nm)
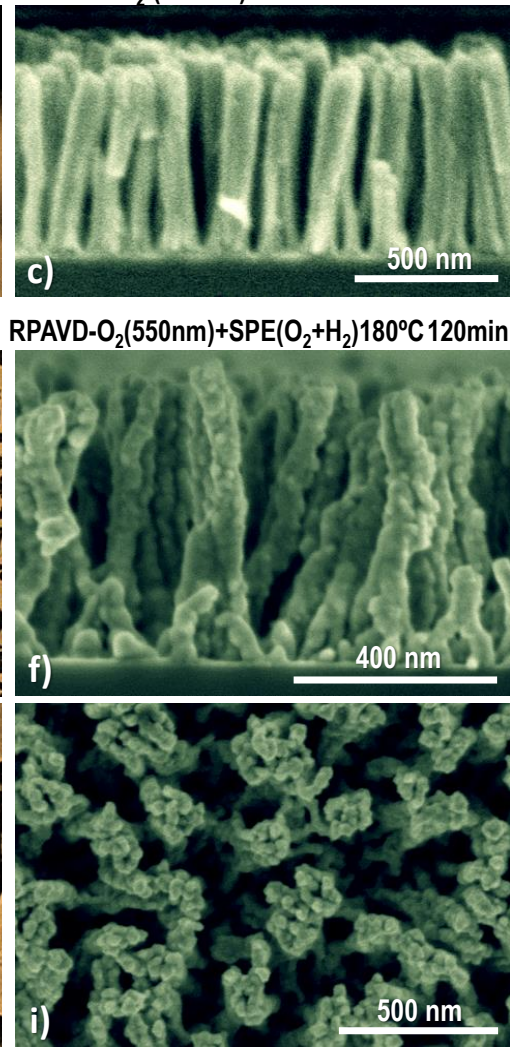

Figure 1. Cross section (a-c, f) and planar views (d-e, g-i) SEM micrographs of several representative samples as labelled. The OPVD, RPAVD-Ar and RPAVD-O $\mathrm{O}_{2}$ are coloured in blue, orange and green, respectively to aid the reader. 
Among them, the morphology of the precursor layer dictated by the deposition method (OPVD, RPAVD-Ar and RPAVD-O ${ }_{2}$ ) is the most important characteristic controlling the final microstructure after the SPE process. Thus, most relevant microstructural changes are detailed in Figure $1 \mathrm{~d}$ ),g) for OPVD, e),h) for RPAVD-Ar and f), i) for RPAVD- $\mathrm{O}_{2}$. Additional SPE treatments are included in the Supporting Information Figures S1-S3. The OPVD films become a highly porous layer of poorly interconnected Pt assemblies leaving part of the substrate uncovered (see also AFM images and profiles in Fig. S4). The percolation, i.e. degree of coated area and connectivity among islands, of the layers increases with the thickness of the precursor film, but nano and micrometric uncoated regions of different sizes are still evident in the film (see Figure S1). A similar behaviour was depicted by RPAVD-Ar post-treated samples (Figs. $1 \mathrm{e}, \mathrm{h}$ ) and S2) with an important difference in the percolation level of the platinum ensembles. As can be observed in Figure $1 \mathrm{e}$ and $\mathrm{h}$ ), percolated Pt sheets are produced by the SPE treatment for intermediate thicknesses $(280 \mathrm{~nm})$, with uncoated regions in the micron range and even below for thicker samples (Fig. S2). In this case, even for thin layers (140 nm, Fig. S2a) the SPE treatment produced more interconnected dendritic features than OPVD. The size of these grains increased with the thickness of the precursor layers producing platinum porous sheets extended in the plane. On the other hand, one common characteristic of the SPE treated samples is the increase of the porosity as can be observed in Figures $1 \mathrm{~d}$ )-i). Although a thorough study of the porosity is beyond the scope of this work, the porosity can be preliminary evaluated by SEM. It is easily observed a relatively high amount of macropores (pores with diameters higher than $50 \mathrm{~nm}$ ) for all the SPE treated samples along with the appearance of mesopores (pores with diameter between 2-50 $\mathrm{nm}$ ) which abundance depends strongly on the SPE treatment. As consequence of such a drastic modification of the microstructure, surface topography and roughness of both OPVD and RPAVD- 
Ar change abruptly as shown in Fig. S4. In the case of the OPVD sample the roughness decrease (from RMS: $19 \mathrm{~nm}$ to 9.5 in $1 \mathrm{x} 1 \mathrm{um}$ images) as the height of the features in the surface is also reduce to the half. RPAVD-Ar samples suffer a drastic increment in the surface roughness (from RMS: $0.5 \mathrm{~nm}$ to $8.9 \mathrm{~nm}$ in $1 \mathrm{x} 1 \mathrm{um}$ ) along to the appearance of agglomerated features with sizes below $200 \mathrm{~nm}$ in diameter on top of the Pt islands. High magnification SEM image in Figure S2 e) addresses the yet highly porosity of the percolated layers showing pores in the range of the mesopores $(2 \mathrm{~nm}<$ $\mathrm{d}<50 \mathrm{~nm}$ ). RPAVD-O 2 sample kept their 1D columnar microstructure after the SPE treatments but increasing greatly the roughness of the surface of such columns due to the formation of platinum nanoparticles (from RMS: $2.9 \mathrm{~nm}$ to $5.9 \mathrm{~nm}$ ). It is interesting to address that although the average column diameter is reduced after the SPE treatment, the size of the nanocolumns agglomerations increases as the number of columns forming the bundles appears to augment (see also Fig. S4). By contrast to the previous samples, the increase of the porosity is mainly due to the enlargement of the initial macropores. Unfortunately, the contribution of the micropores (pores with diameters below $2 \mathrm{~nm}$ ) cannot be evaluated since this type of pores is very small to be observed by SEM. Finally, it is worth to mention the reduction in thickness of all the post-treated samples when compared with the as-grown layers (Fig.1 and S3).

Glancing Angle X-Ray Diffraction (GAXRD) analysis was performed in order to evaluate the crystalline condition of the samples. Figure 2 gathers GAXRD patterns from the precursor layers, showing a pronounced peak at low angles alongside lower intensity peaks between $15^{\circ}$ and $30^{\circ}$ for the sublimated thin film (OPVD (550nm)) according to its polycrystalline character. ${ }^{19,27}$ Patterns acquired for the samples grown by RPAVD presented no peaks independently on the plasma gas composition. This result is in good agreement with the smooth and homogeneous microstructure depicted by the samples RPAVD-Ar, similar to the standard nanocomposite organic thin films 
deposited by this technique. ${ }^{22-25}$ By contrast, all the SPE treated samples (regardless the deposition method) showed well defined peaks corresponding to the planes (111), (200), (220), (311) and (222), which are characteristic of the fcc structure of Pt. ${ }^{30}$
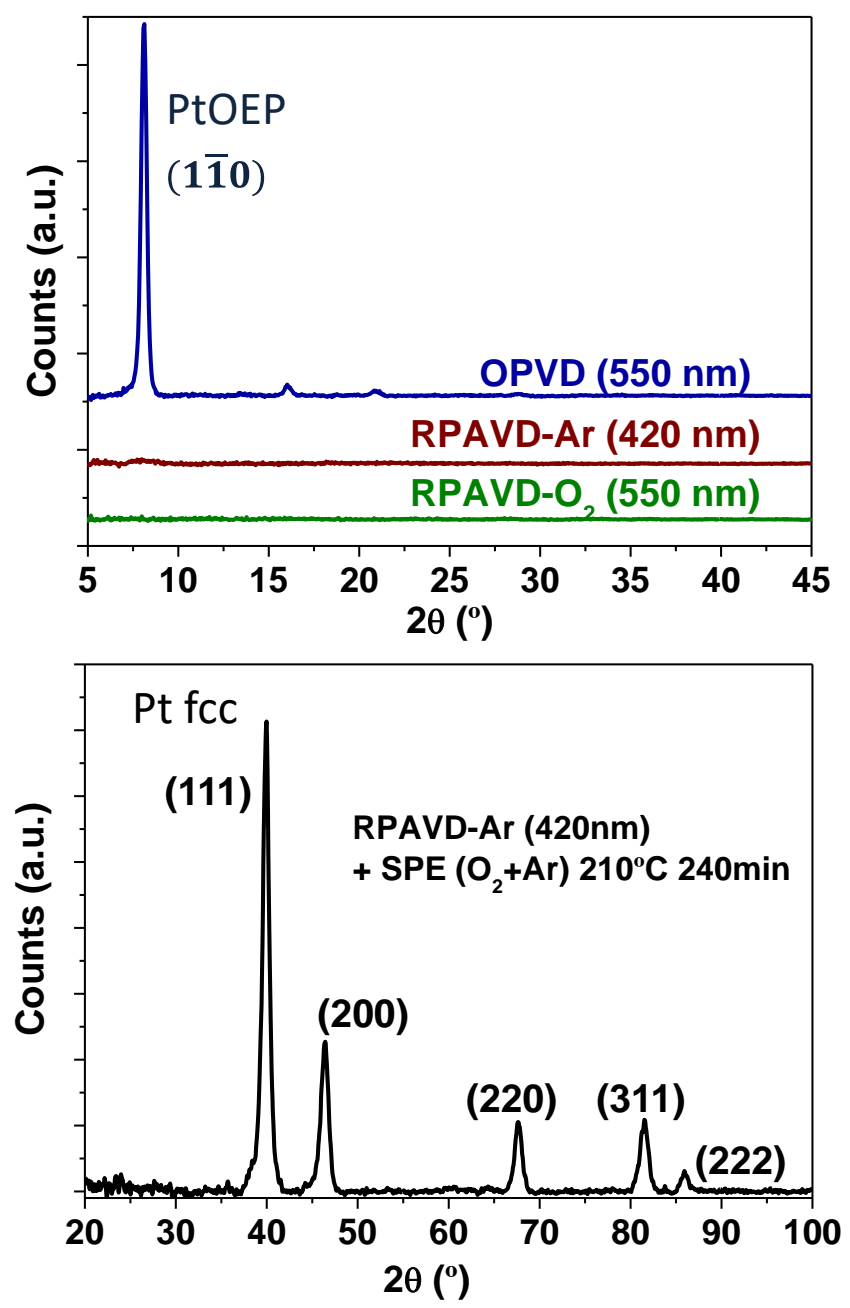

Figure 2. Top) GAXRD of the precursor layers formed by OPVD and RPAVD under argon and oxygen plasma gases conditions. Bottom) GAXRD pattern of a platinum layer formed after plasma etching of a RPAVD-Ar (420nm) precursor film.

In order to follow the decomposition of the PtOEP into Pt, in situ experiments by X-ray Photoelectron Spectroscopy (XPS) were carried out. In these experiments, a thin layer (40 $\mathrm{nm})$ of PtOEP was sublimated and plasma post-treated $\mathrm{SPE}\left(\mathrm{Ar}+\mathrm{O}_{2}\right)$ at $175^{\circ} \mathrm{C}$ in a prechamber attached to the XPS analysis chamber (See Experimental Section). Figure 3 gathers the main XPS peaks acquired during such experiments. Full decomposition of the platinum 
porphyrin was achieved with treatments longer than 20 minutes, pointed out by the vanishing of the N1s peak. Figure 3 shows the shift of the Pt4f peaks towards lower binding energies values in good agreement with the formation of $\operatorname{Pt}(0) .{ }^{31}$ This experiment also provides the binding energy position of the $\mathrm{Pt} 4 \mathrm{f} 7 / 2$ peak when platinum is coordinated in the PtOEP molecule $(\sim 72.2 \mathrm{eV})$. This value will be critical for the elucidation of the oxidation state of the platinum as observed in the ex situ experiments. Interestingly, the observed increasing of the $\mathrm{O} 1 \mathrm{~s}$ peak is also compatible with the partial oxidation of the molecule and the exposition of open areas of the silicon substrate (see purple line in the O1s peak in Fig. 3), which reveals the silicon oxide native layer.
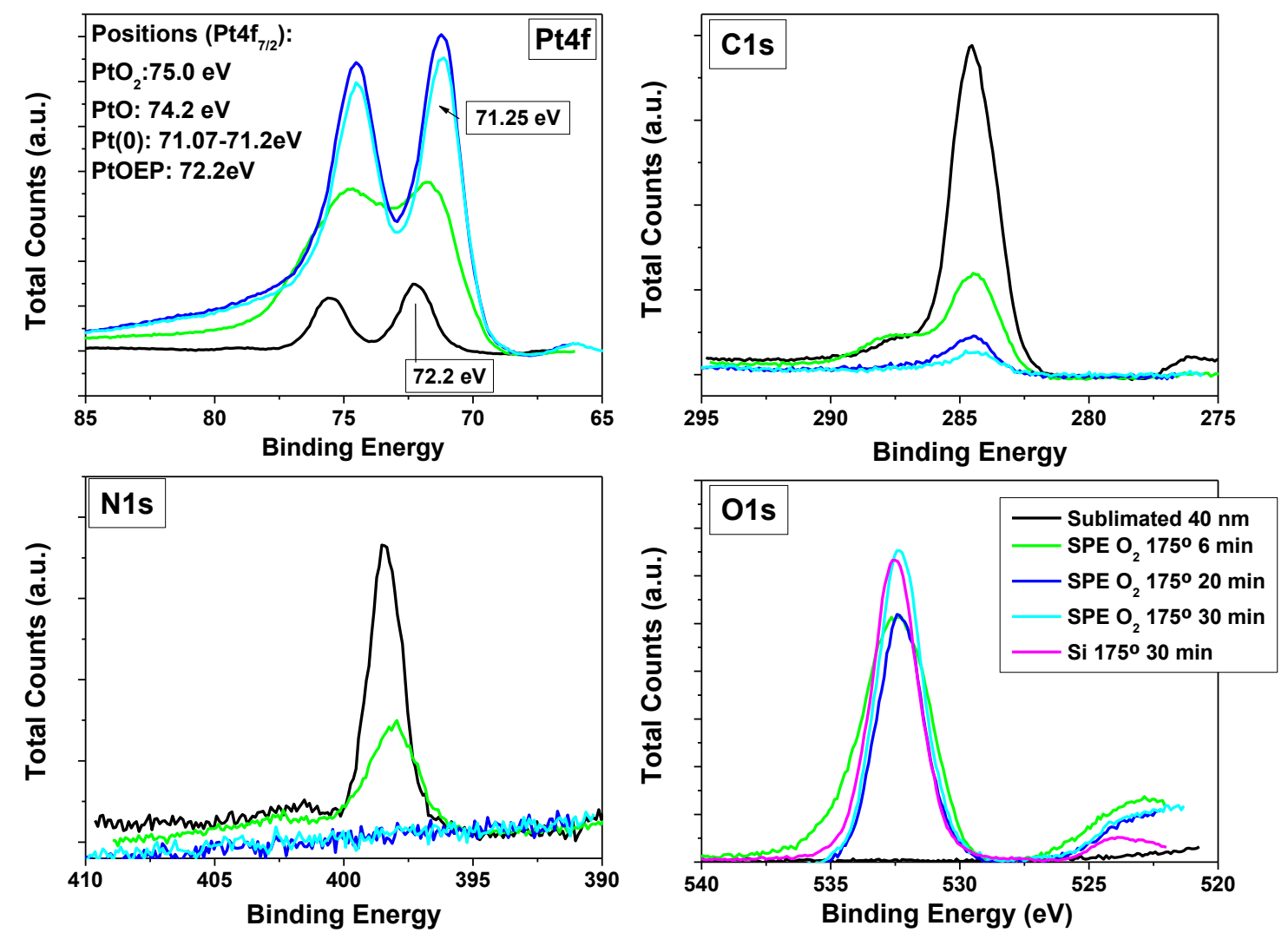

Figure 3. In situ XPS experiments following the soft plasma etching in $\mathrm{O}_{2}+\mathrm{Ar}(300 \mathrm{~W})$ at $175^{\circ} \mathrm{C}$ of the PtOEP OPVD film (40 nm). Surface was sequentially exposed to plasma. 
Table 1. Atomic percentage of the elements (also the different Pt oxidation environments deduced from Figure S6) present in the samples.

\begin{tabular}{|c|c|c|c|c|c|c|c|}
\hline Sample & $\begin{array}{c}\mathrm{Pt}(0) \\
\%\end{array}$ & $\begin{array}{c}\mathrm{Pt} \% \\
(\mathrm{PtOEP})\end{array}$ & $\begin{array}{l}\mathrm{Pt} \% \\
(\mathrm{PtO}- \\
\left.\mathrm{PtO}_{2}\right)\end{array}$ & $\mathrm{O} \%$ & $\mathrm{~N} \%$ & $\mathrm{C} \%$ & Si \% \\
\hline OPVD (550nm) & - & 2.9 & - & 4.9 & 6.7 & 85.5 & - \\
\hline RPAVD-Ar (280nm) & - & 2.4 & - & 18.8 & 7.1 & 71.7 & - \\
\hline RPAVD-O $_{2}(200 \mathrm{~nm})$ & - & 5.8 & 7.6 & 47.6 & 1.7 & 27.9 & 9.4 \\
\hline $\begin{array}{l}\text { RPAVD-Ar }(280 \mathrm{~nm}) \\
+\mathrm{SPE}\left(\mathrm{O}_{2}+\mathrm{Ar}\right) 180^{\circ} \mathrm{C} \\
120 \mathrm{~min}\end{array}$ & 5.7 & - & 3.5 & 46.2 & 1.0 & 14.4 & 29.2 \\
\hline $\begin{array}{c}\text { RPAVD-Ar (280nm) } \\
+\mathrm{SPE}\left(\mathrm{O}_{2}+\mathrm{Ar}\right) 180^{\circ} \mathrm{C} \\
120 \mathrm{~min}+\text { Anneal }\left(\mathrm{H}_{2}+\mathrm{Ar}\right)\end{array}$ & 9.6 & - & - & 41.9 & 1.3 & 18.5 & 28.7 \\
\hline $\begin{array}{c}\text { RPAVD-O }{ }_{2}(200 \mathrm{~nm})+\mathrm{SPE} \\
\left(\mathrm{Ar}+\mathrm{O}_{2}\right) 180^{\circ} \mathrm{C} 140 \mathrm{~min}+ \\
\text { Anneal }\left(\mathrm{H}_{2}+\mathrm{Ar}\right)\end{array}$ & 11.1 & - & - & 35.1 & 1.5 & 30.7 & 21.6 \\
\hline
\end{tabular}

It can be concluded that the porphyrin was completely decomposed after SPE $\left(\mathrm{Ar}+\mathrm{O}_{2}\right)$ at mild temperature, giving rise to the formation of platinum metal clusters. However, equivalent experiments at room temperature demonstrated that prolonged treatment did not effectively decompose the organic counterpart. ${ }^{27,28}$ This latter result might be interesting for determined final applications since nitrogen-platinum bonds have been identified as highly active catalytic sites. ${ }^{8}$

In order to assess the role of the growth conditions and post-treatments on the final chemical composition, we analysed the as-grown samples before and after SPE by means of ex situ XPS (see Table 1 and Fig. S5-S7). The three first rows on Table 1 correspond to the as-grown samples comparing the OPVD, RPAVD-Ar and RPAVD$\mathrm{O}_{2}$ layers. Figure $\mathrm{S} 5$ and $\mathrm{S} 6$ show correspondently the general spectra and the deconvolution of the Pt $4 \mathrm{f}$ peaks to determine the composition of the samples and the oxidation state of the platinum and distinguish between $\mathrm{Pt}(\mathrm{Pt} 4 \mathrm{ff} / 2 \sim 71.0-71.2 \mathrm{eV})$, PtOEP $(\mathrm{Pt} 4 \mathrm{f} 7 / 2 \sim 72.2 \mathrm{eV}), \mathrm{PtO}(\mathrm{Pt} 4 \mathrm{f} 7 / 2 \sim 74.2 \mathrm{eV})$ and $\mathrm{PtO}_{2}(\mathrm{Pt} 4 \mathrm{f} 7 / 2 \sim 75 \mathrm{eV}){ }^{31}$ Results in Table 1 indicate that after the SPE treatments, the metal organic layers got 
richer in metallic platinum as the percentage of nitrogen and carbon decreases. The complete conversion into metallic platinum required post-annealing under $\mathrm{H}_{2}+\mathrm{Ar}$ (Fig. S4 e)-f)). Concerning the $\mathrm{C} 1 \mathrm{~s}, \mathrm{O} 1 \mathrm{~s}$ and N1s signals (Figure S7), their evolutions are those ones expected for the oxidation and combustion of the organic chains in the PtOEP molecule. Thus C1s and N1s signals, initially single and quite symmetrical, develop high binding energy tails along with the plasma treatments, due to the formation of amide, carbonyl and carboxyl groups (see also Fig. 3). In addition, the N1s intensities decrease quickly as the duration of the plasma treatment rise. Oxygen in the OPVD sample is very likely related to a slight surface oxidation of the porphyrin and water adsorption as consequence of exposure to ambient conditions. Note that the in situ deposited film does not contain oxygen (Fig. 3). For the rest of the samples, there are several additional contributions to the observed oxygen content. On the one hand, the oxygen linked to the silicon oxide substrate exposed after the SPE $\left(\mathrm{O}_{2}+\mathrm{Ar}\right)$ treatments and to the partial carbon oxidation. On the other hand, it can be attributed to the formation of platinum oxide under RPAVD- $\mathrm{O}_{2}$ conditions and after SPE $\left(\mathrm{O}_{2}+\mathrm{Ar}\right)$ treatment (see Fig. S6-S7).

Although the complete characterization of the plasma is out of the scope of this article, the role of the different gases during the growth can be understood in terms of plasma reactivity. In the case of $\mathrm{Ar}$, the main effect of the plasma consists on molecular bombardment. This bombardment produces partial fragmentation of the PtOEP molecules and radicals that induce their binding with the surrounding molecules in a type of polymerization generating smooth and highly conformal layers. ${ }^{24}$ As demonstrated elsewhere for organic functional molecules, the reactor geometry and plasma pressure conditions allow the formation of the nanocomposite films maintaining a percentage of integer molecules..$^{24,25,29}$ On the other hand, the reactivity of oxygen plasma is significantly higher. The plasma produces a partial oxidation of 
the molecules (see XPS data) that lose the optical spectral features of the PtOEP as we will discuss in the next section (see Figure $4 \mathrm{e}$, where the bands corresponding to the PtOEP at 380, 510 and $530 \mathrm{~nm}$ are not present). The particular columnar morphology of the RPAVD- $\mathrm{O}_{2}$ may be attributed to the low mobility and high sticking coefficient of the oxidized precursor fragments. Under these conditions, the first ad-molecules would form islands nuclei favouring a growth mechanism strongly controlled by shadowing effects. In this way the subsequent fragments of precursor arriving to the surface would form the characteristic columnar growth. Similar arguments have been successfully applied to the growth of nanoporous metal oxide thin films by PECVD using organometallic precursors. ${ }^{[32-34]}$ Oxygen-Argon and Oxygen-Hydrogen plasmas are commonly used for surface polymer modifications and to etch organic films by the

production of volatile organic compounds that are pumped out of the reactor. ${ }^{35-36}$ In our system, the process is enhanced with mild temperatures leading to the complete removal of the organic components of the films to obtain pure Pt supported nanostructures. The results indicate the $\mathrm{O}_{2}+\mathrm{H}_{2}$ mixtures at mild temperatures are the most efficient to etch relatively thick films in our experimental conditions.

\subsection{Optical properties}

Figure 4 shows the UV-VIS-NIR transmittance spectra of some representative samples deposited on fused silica substrates and post-treated under different SPE conditions. The figure shows the curves for the as-grown (OPVD (a), RPAVD-Ar (c) and RPAVD-O 2 (e) of two different thicknesses) and SPE post-treated (b,d,f) samples, where it can be noted the ample variety in the transmittance depending on the experimental conditions. It is worth to mention that the post-annealing in $\mathrm{H}_{2}+\mathrm{Ar}$ gases did not significantly affect the optical properties of the samples (see Fig. S8) and therefore such label does not appear in the optical study for the sake of simplicity. 
As-grown OPVD samples present the characteristic bands of the PtOEP molecule. ${ }^{37}$ SPE $\left(\mathrm{O}_{2}+\mathrm{Ar}\right)$ treatment at room temperature of the OPVD samples did not decompose the porphyrin efficiently as already mentioned regarding the in situ XPS experiments, even for layers thinner than $190 \mathrm{~nm}$ (see Fig. S5). To fully decompose the organic matrix is necessary to increase the SPE temperature above $160^{\circ} \mathrm{C}$. In fact, highly flat transmission curves were obtained for all the SPE $\left(\mathrm{O}_{2}+\mathrm{Ar}\right)$ treatments at such temperature. The initial sample thickness before the plasma treatments (i.e. the amount of metal in the sample) determines the transparency of the SPE post-treated layers (Figure 4b). It is worthy to mention the extremely constant transmittance of these samples, that are comparable to results presented in the literature for complex metal and metal/CNTs networks. ${ }^{11}$ The sample thickness also dictates the temperature and duration required in the SPE treatment for a full mineralization of the samples. Thus for the thickest OPVD sample, a more intensive SPE treatment under $\left(\mathrm{H}_{2}+\mathrm{O}_{2}\right)$ and higher temperature and time $\left(180^{\circ} \mathrm{C}, 120 \mathrm{~min}\right)$ were necessary to reach the flat spectrum shown of Fig. 4b) (pink line).

Figure $4 \mathrm{c}$-d) summarizes the main results regarding the optical characterization of the RPAVD-Ar as-grown (c) and post-treated samples (d). As expected from previous results of RPAVD of organic molecules, ${ }^{22-25}$ samples deposited under these plasma conditions contained integer PtOEP molecules along with small moieties formed after partial plasma fragmentation of the molecule. For thinner layers the characteristic bands presented a weaker absorption in good agreement with results extracted for OPVD samples. SPE $\left(\mathrm{O}_{2}+\mathrm{Ar}\right)$ treatments of the samples induced the mineralization of the PtOEP vanishing the organic-related bands and giving rise to constant transmittance spectra. In this case, the SPE treatment required higher temperatures and extended times in order to produce the full decomposition of the molecule (see pink line in Fig. 4d), a result that is related to the dense microstructure of the films. 
Increasing the treatment duration decreased the transmission, reaching a plateau for long times (compare black and red lines), which is taken as an indication of the full mineralization of the sample. Again, for the thickest sample an SPE treatment with $\left(\mathrm{H}_{2}+\mathrm{O}_{2}\right)$ and higher temperature and time $\left(180^{\circ} \mathrm{C}, 120 \mathrm{~min}\right)$ were necessary to complete the mineralization (dark yellow line).
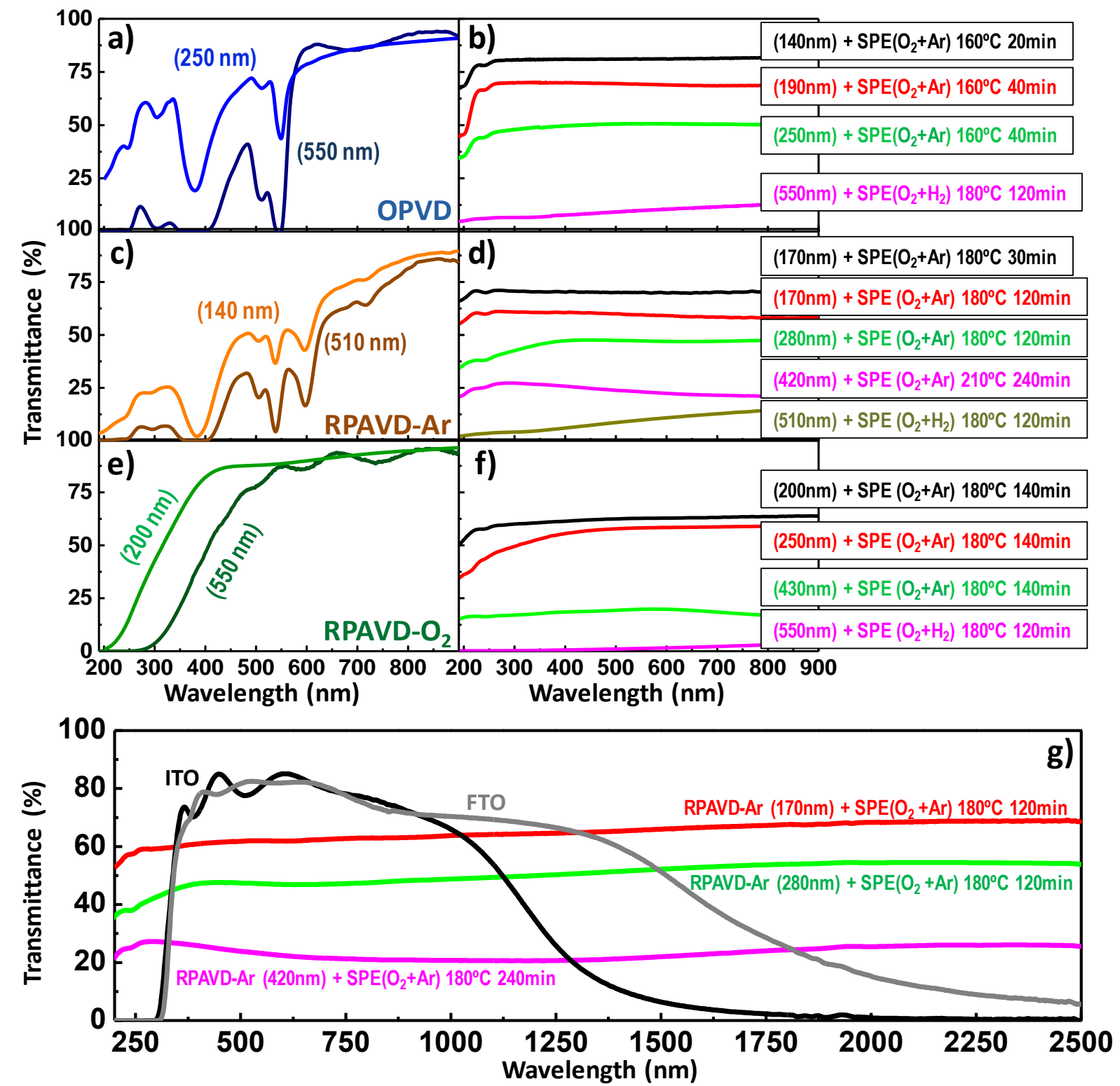

Figure 4. a-f) UV-VIS spectra of OPVD (a), RPAVD-Ar (b) and RPAVD-O ${ }_{2}$ (c) and the corresponding plasma treated samples as labelled. All the treated samples were characterized after a post-annealing treatment in $\mathrm{H}_{2} / \mathrm{Ar}$ at $135^{\circ} \mathrm{C}$. g) UV-Vis-NIR $(200-2500 \mathrm{~nm})$ spectra of 
selected RPAVD-Ar plasma treated samples in comparison to ITO and FTO reference layers showing the highly flat transmittances in this range of the Pt layers.

Figure 4 e,f) presents the UV-Vis spectra corresponding to as-grown (e) and posttreated (f) RPAVD-O ${ }_{2}$ samples. It is important to stress the peculiar behaviour of the as-grown RPAVD-O ${ }_{2}$ in contrast to OPVD and RPAVD-Ar samples. In this case, the spectra for the as-grown sample presented an absorption edge below $400 \mathrm{~nm}$ that strongly depends on the sample thickness (Fig. 4 e). These absorption features are in concordance with the XPS data regarding the formation of platinum oxides. However, the absence of molecular bands in the UV-Vis spectra appears to be in contradiction with the percentage obtained by XPS of Pt yet forming PtOEP. This is very likely due to partial damaging of porphyrin molecules under the remote oxygen plasma affecting to the electron conjugation but keeping the $\mathrm{Pt}$ in a similar nitrogen environment. Once the SPE treatments were carried out on the samples, the transmittance spectra presented a similar behaviour than for OPVD and RPAVD-Ar post-treated samples. In this case, a substrate temperature about $180^{\circ} \mathrm{C}$ allowed the conversion into platinum of the metal-organic porphyrin. It is worth to mention that the thicker sample treated with SPE $\left(\mathrm{H}_{2}+\mathrm{O}_{2}\right)$ (pink colored line) presented the lowest transmission of all the analysed samples with a value below $5 \%$ in the full UV-VIS range increasing slightly with the wavelength.

In summary, the methodology reported herein allows to fabricate neutral density filters with an adjustable optical density dictated by the initial thickness of the film and the soft plasma etching treatment. The variation of the optical density values of the different SPE treated samples is related to the empty volume of the films, which is intimately related to the porosity. The control over the optical density and the deviation from the ideally flat filter for the samples presented in Figure 4 are shown in Figure S9. Very small mean deviations below ca. 
$4 \%$ are obtained for all the cases, indicating the good quality of the fabricated filters. The representation and fitting of the optical density $(\mathrm{OD}=-\log (\mathrm{T} / 100))$ vs Thickness of the original thin film is also presented in Figure S9, allowing to accurately design the filter according to the desired OD. Moreover, the RPAVD-Ar showed the flattest profile over a wide wavelength range. The curves in the 200-2500 $\mathrm{nm}$ range for three representative RPAVD-Ar samples are shown in Figure $3 \mathrm{~g}$ ). The figure also shows the spectra of commercial ITO and FTO, which block the transmission of light above 1500 and $2200 \mathrm{~nm}$, respectively, whereas the samples prepared with the methodology reported do not show any significant variation in this spectral range. It can be observed a very flat profile with mean deviations between $2.4-3.2 \%$, showing the good behaviour of the samples. This together to the high Pt stability open a new way for the fabrication of wide range neutral optical filters.

\subsection{Electrical properties}

The Pt nanostructures generated by the reported plasma-assisted methodology were conceived for applications which require electrical conductivity. In-plane electrical conductivity measurements were performed by the four-point probe method on the films deposited on fused silica and onto preformed electrodes separated $100 \mu \mathrm{m}$ (see Experimental Section). As it can be inferred from the SEM analysis, the samples do not present a good laminar conductivity due to the gaps observed for the OPVD and RPAVD-Ar samples and to the nanocolumnar microstructure in the RPAVD-O 2 case. The extrapolated sheet resistances and a detailed description of the measurements are presented in the ESI Section S10. It is worth to mention that for the electrical behaviour (by contrast to the optical), the reduction treatment (post annealing in $\mathrm{H}_{2}+$ Ar) leads to an improvement of $50 \%$ in the conductivity of the samples (Fig. S10). A clear and evident tendency can be obtained for all the samples: the resistances decreased as the thickness of the sample increased. The highest values of conductivity 
correspond to very thick samples post-treated under $\mathrm{H}_{2}+\mathrm{O}_{2}$ plasma. On the other hand, the thicker samples with lower sheet resistances depicted very low transmittances of light (see transmittance value in Table S1).

The fabrication of transparent metallic conductors requires two conditions, a very high porosity and a very high lateral connectivity. For this purpose, RPAVD-Ar samples presented the lower resistances with reasonably high transmissions of light. For example, RPAVD-Ar of $280 \mathrm{~nm}$ thickness treated with $\mathrm{SPE}\left(\mathrm{O}_{2}+\mathrm{Ar}\right), 180^{\circ} \mathrm{C}, 120 \mathrm{~min}$, presented a sheet resistance better than $1200 \Omega / \square$ and a relatively high transmittance value of $47 \%$ (see section S10). Those resistance values, although far from the obtained for transparent conductive oxides such as ITO $(\mathrm{RS}=5-10 \Omega / \square$, Transmittance@ 500=80-85\%), were low compared to Pt thin films from the bibliography. Thus, similar transparencies of ca. $50 \%$ have been reported for Pt films with 4-5 nm thickness presenting a RS value higher than $30000 \Omega / \square .{ }^{38,39}$ Although better conductive transparent films have been recently reported for Pt-coated fibers or Pt-nanomesh, ${ }^{17,18}$ the methodology developed here is promising for specific catalytic applications such as counter electrodes in solar cells. In this case, the Pt is crucial as catalyst for the reduction of a liquid electrolyte $\left(\mathrm{I}_{3}^{-} / \mathrm{I}^{-}\right)$which requires a good transversal conductivity. Looking to this final application, transversal conductivity was measured by conducting atomic force microscopy (C-AFM) on samples depicting columnar microstructure (i.e. formed under RPAVD-O $\mathrm{O}_{2}$ conditions) deposited onto a gold thin film. This technique makes use of a metallic tip in contact mode, thus usually obtaining slightly distorted topographic images consisting on a convolution of tip and surface. The advantage of this technique is that allows the simultaneous acquisition of topographic images (Fig. 5 a) and conductivity maps (Fig. 5 b). The topography image of the sample shows features equivalents to those observed by SEM (Figure S2) and by AFM (Figure S4) with bundles of columns with diameters in the order of $100 \mathrm{~nm}$. 
Please, note that a more accurate topographic image is shown in S4 since AFM measurement modes (tapping in AFM and contact for C-AFM) and tip diameters are different in Figures 5 and S4. Nevertheless, conductivity map acquired at 0.01V (Fig. 5b) shows a fairly good agreement with the topography image (a), where a high current of ca. $10 \mathrm{nA}$ is obtained at the apex of the columns. The IV curves at different locations of the image in Fig. 5 b) are shown in c). The curves recorded at the brightest areas (apex of the columns, solid lines) range between several nanoamperes of current flowing through one of these columns to almost negligible values in between columns on the darker areas (dashed lines). The straight behaviour of the I-V curves indicates an ohmic contact (Pt/Ir tip-Pt nanocolumns-Au thin film) as expected for metal-metal joints.

Although the C-AFM provides real current measurements, the different joints in the system introduce serial ohmic resistances that lower the recorded current, making hard to extrapolate real resistivity. To have a clearer idea about the resistivity of the RPAVD-O ${ }_{2}$ samples, a commercial ITO sample with a given resistivity of $4 \Omega / \square$ was measured by C-AFM (see the corresponding I-V curve in Fig. 5d) for comparison as red dashed curve, multiplied by 10). It can be observed the low current observed for the ITO sample, which is below $0.1 \mathrm{nA}$ at $0.01 \mathrm{~V}$ (100 times lower than at the apex of the columns, solid lines). Although in this case the I-V curve for ITO showed a rectifying behaviour for higher voltages (not shown), it is clear that the RPAVD- $\mathrm{O}_{2} \mathrm{Pt}$ nanocolumnar films have a very high transversal conductivity and therefore are an optimum candidate for specific applications such as counter electrodes in DSCs as shown in the next section. 


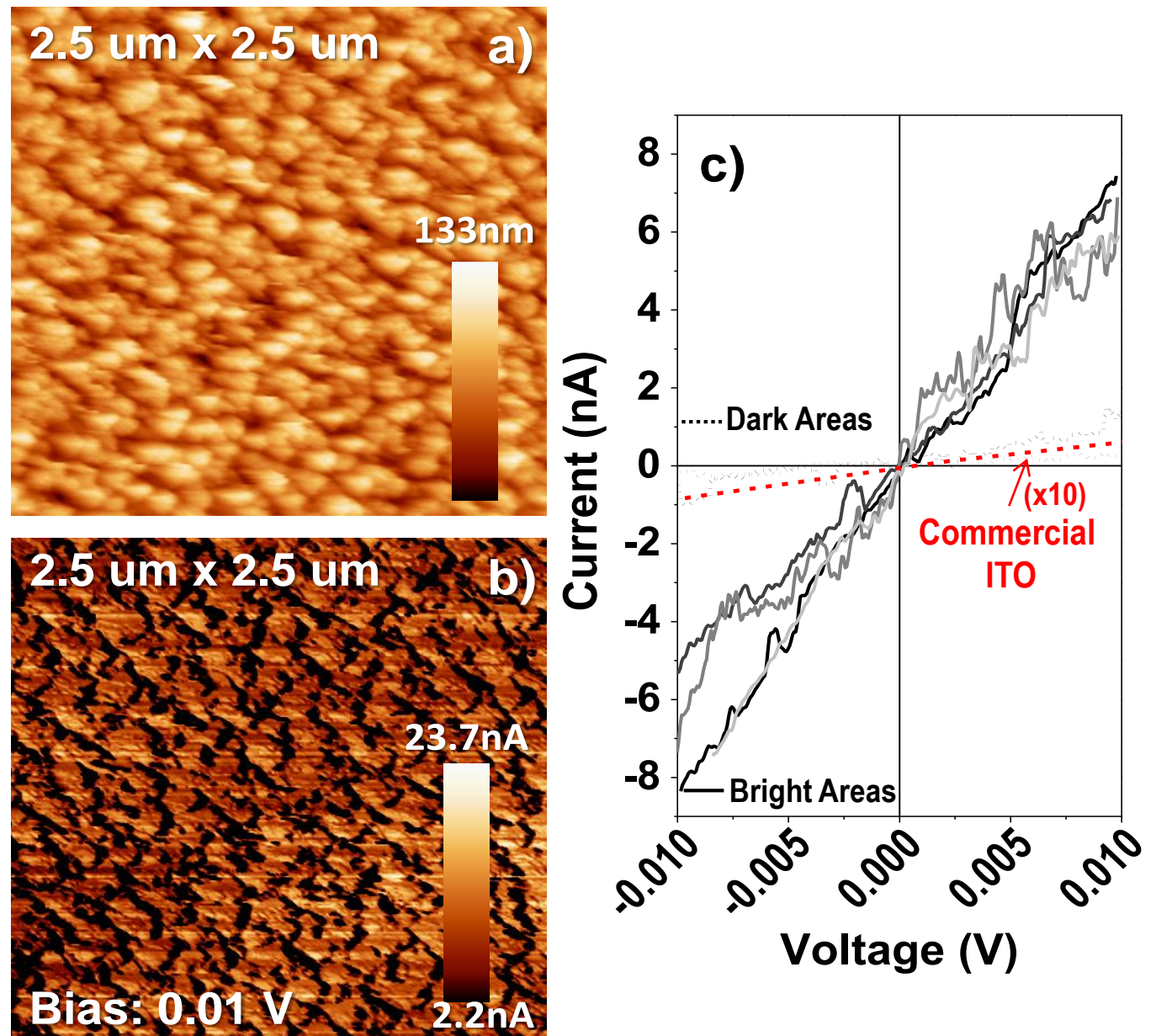

Figure 5. Topography AFM (a-b) images acquired at two different scales in the nanocolumnar "RPAVD-O $2(200 \mathrm{~nm})+\mathrm{SPE}\left(\mathrm{Ar}+\mathrm{O}_{2}\right) 180^{\circ} \mathrm{C} 140 \mathrm{~min}$ " sample. d) I-V curves measured with the AFM tip placed on a position with a maximum (solid lines) and minimum (dotted lines) conductivity. The curve obtained for a commercial ITO sample is included for comparison (red dashed line, multiplied by 10).

\subsection{D and 2D transparent catalytic Platinum layers as counter electrodes in Solar Cells}

An efficient counter electrode for DSC must have a very high catalytic surface area and therefore the Pt nanostructured films presented here are ideal candidates for this purpose. In this section we will show the implementation in a real DSC device of 1D RPAVD-O $2(500 \mathrm{~nm})$ and 2D (RPAVD-Ar (420nm), both post treated with $\operatorname{SPE}\left(\mathrm{Ar}+\mathrm{O}_{2}\right) 210^{\circ} \mathrm{C}, 240 \mathrm{~min}$ and Anneal $\left(\mathrm{Ar}+\mathrm{H}_{2}\right)$. Thus, DSCs using counter 
electrodes with the catalytic platinum nanostructures reported have been fabricated and compared to classical FTO counter electrodes coated with commercial Platisol (Solaronix) (for further details see experimental section). In order to gather additional information about the influence of these platinum electrodes on the performance of DSCs, a commercial low-viscosity solvent electrolyte based on acetonitrile and valeronitrile (AN50, Solaronix), and a high-viscosity pure ionic (Pyrrolidinium, Solvionic) solvent electrolyte were tested. Figure 6 shows the I-V curves for the three kinds of counter electrodes used in the study and for the two distinct electrolytes employed. Table 2 presents the different parameters obtained for the fabricated solar cells with electrolyte AN50. Along with the higher photocurrent and photovoltage given for the vacuum deposited platinum, a higher fill factor was observed for these cells compared to the standard Platisol ones. These facts suggest that our electrodes depict a better catalytic behaviour, allowing a faster regeneration of iodide ions at the counter electrode/electrolyte interface. By contrast, the cells prepared with the highviscosity electrolyte (Pyr 100\%, see extracted photovoltaic parameters in Table 2) present minor differences in the photovoltaic parameters for the different counter electrodes. Only the 1D counter-electrodes exhibited a slight superior efficiency (on average) due to a higher photocurrent. The smaller efficiencies observed for Pyr $100 \%$ are expected for such a higher-viscosity formulation. ${ }^{39-42}$ The potential faster reduction of tri-iodide by our electrodes is expected to be more noticeable in cells with a higher photocurrent, since in this case a more rapid regeneration is required. Hence, if triiodide cannot diffuse fast enough to the counter electrode, it really does not matter whether its reduction rate is further increased because the reaction is diffusion-limited. Electrochemical Impedance Spectroscopy (EIS) was carried out to get further insight of the behaviour of the electrodes. Figure 6 b) and c) present the Nyquist plots for the DSCs fabricated with AN50 and Pyr 100\%, respectively. It can be seen in Fig. 6 b) that 
Platisol gave rise to the lowest series resistance (onset of the impedance curve at high frequencies) whereas the platinum RPAVD-Ar network originated the largest. However, the width of the first semicircle, corresponding to the Pt/electrolyte interface ${ }^{43}$ decreased in the order Platisol $>$ nanorods $\left(\right.$ RPAVD-O $\left.{ }_{2}\right)>$ network (RPAVD- $\mathrm{O}_{2}$ ). This is indicative of a decreasing charge transfer resistance at the Pt/electrolyte interface and is in agreement with the previously suggested higher catalytic performance, the faster regeneration of the redox couple and the higher photocurrents observed. It also contributes to a decrease of the voltage drop occurring at the same interface, which would explain the larger Voc.

Figure S11 presents the chemical capacitance extracted from EIS analysis at different potentials. Figure S12 depicts the corresponding circuit diagram. The EIS results show that the capacitance is not altered by the different counter electrodes, which indicates that, probably as a consequence of using the same electrolyte composition, no significant shifts of the conduction band are occurring. Therefore, taking into account the following equation: ${ }^{42,43}$

$$
\tau_{n}=R_{r e c} C_{\mu} \quad \text { Eq. } 1
$$

The electron lifetime is mainly determined by the electron recombination resistance at the $\mathrm{TiO}_{2}$ /electrolyte interface. As shown in Figure S11, recombination resistances are slightly higher for the RPAVD-Ar platinum network and lower for Platisol. The lowering of the recombination rate can be explained by the lower tri-iodide concentration due to the faster regeneration of the redox couple for platinum grid counter electrodes. Hence, the beneficial effect of the new platinum films is twofold: on the one hand the higher catalytic activity allows a faster regeneration at the counter electrode, and secondly, as a consequence, recombination with tri-iodide acceptors is slower. This produces a simultaneous enhancement of both Jsc and Voc. 
In summary, catalytic platinum nanorods and percolated layered networks have demonstrated a superior photovoltaic performance when used as counter electrodes compared to the Platisol commonly used in DSCs when a low-viscosity electrolyte was employed.

a)

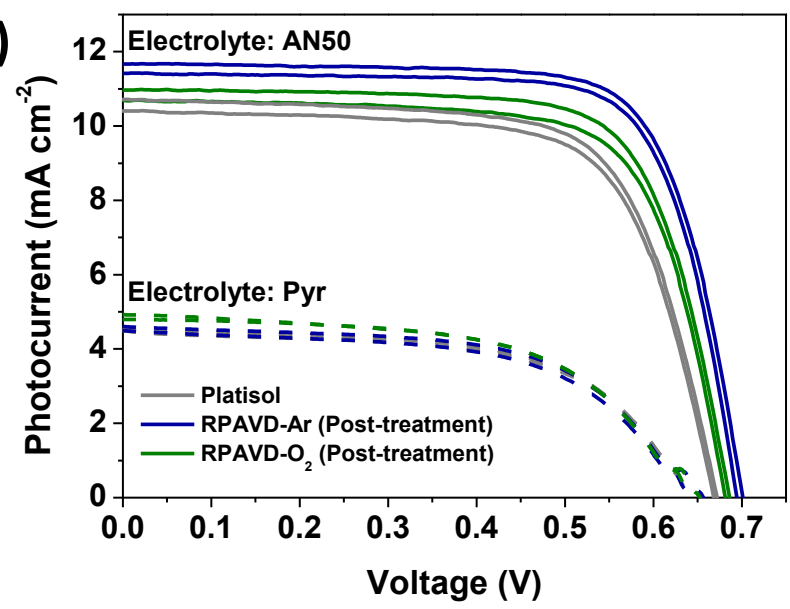

b)
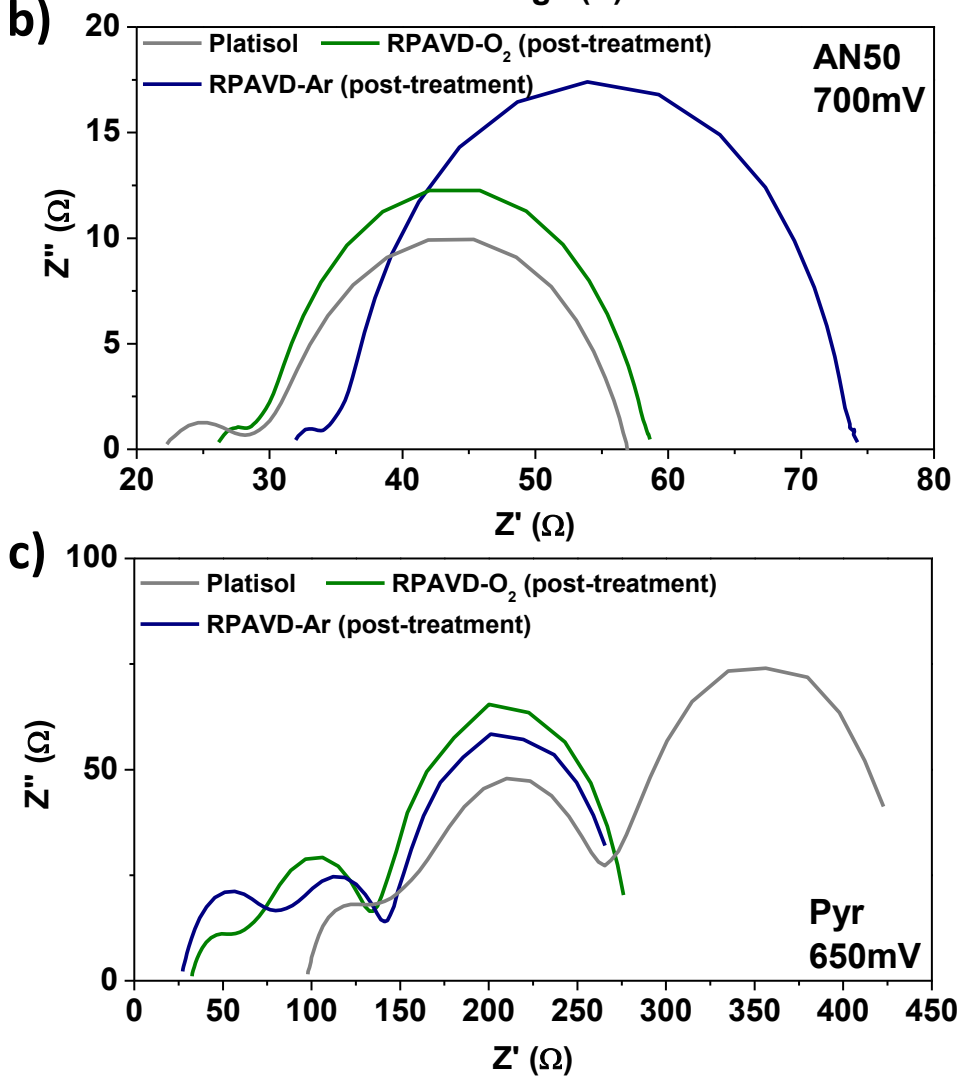

Figure 6. a) I-V curves for the different counter electrodes and electrolytes employed. b,c)

Nyquist plot of DSCs with N50 (b) and Pyr (c) electrolytes. The voltages used for the measurements are indicated in the respective figure. 
Table 2. Characteristic photovoltaic parameters for electrolytes AN50 and Pyr 100\%. Given values are obtained after characterization of two cells for each condition.

\begin{tabular}{|c|c|c|c|c|}
\hline $\begin{array}{l}\text { Electrolyte } \\
\text { AN50 }\end{array}$ & $\mathrm{Jsc}\left(\mathrm{mA} \cdot \mathrm{cm}^{-2}\right)$ & Voc $(\mathrm{mV})$ & Fill Factor & $\eta(\%)$ \\
\hline Platisol & $10.55 \pm 0.15$ & $669.5 \pm 1.5$ & $68.95 \pm 0.05$ & $4.90 \pm 0.10$ \\
\hline RPAVD-Ar (post-treatment) & $11.5 \pm 0.1$ & $697 \pm 3$ & $74.05 \pm 0.50$ & $5.95 \pm 0.05$ \\
\hline RPAVD-O ${ }_{2}$ (post-treatment) & $10.65 \pm 0.15$ & $683 \pm 2$ & $71.80 \pm 0.40$ & $5.25 \pm 0.15$ \\
\hline $\begin{array}{l}\text { Electrolyte } \\
\text { Pyr } 100 \%\end{array}$ & $\mathrm{Jsc}\left(\mathrm{mA} \cdot \mathrm{cm}^{-2}\right)$ & Voc $(\mathrm{mV})$ & Fill Factor & $\eta(\%)$ \\
\hline Platisol & $4.55 \pm 0.05$ & $664 \pm 1$ & $57.7 \pm 0.3$ & $1.70 \pm 0.01$ \\
\hline RPAVD-Ar (post-treatment) & $4.55 \pm 0.05$ & $648 \pm 4$ & $57.55 \pm 0.55$ & $1.75 \pm 0.05$ \\
\hline RPAVD-O ${ }_{2}$ (post-treatment) & $4.8 \pm 0.1$ & $648 \pm 1$ & $56.85 \pm 0.75$ & $1.80 \pm 0.01$ \\
\hline
\end{tabular}

\section{Conclusion}

We have developed a full vacuum/plasma assisted methodology for the fabrication of platinum nanostructured multifunctional layers. RPAVD-Ar conditions produce a compact continuous layer forming a homogeneous and smooth film containing integer PtOEP molecules embedded in a polymeric-like matrix. By contrast, the RPAVD-O ${ }_{2}$ conditions produce a completely different microstructure consisting in nanocolumns composed mainly of platinum oxides. As far as we know this is the first time that $\mathrm{PtO} / \mathrm{PtO}_{2}$ formation by plasma processing of platinum porphyrin has been reported. The nanocolumns formation under RPAVD- $\mathrm{O}_{2}$ is especially appealing giving its particular microstructure, high homogeneity in composition and interesting optical properties (i.e. non-dispersive structures and thickness dependent absorption edge within the near/medium UV range). 
In this methodology, a Soft Plasma Etching process is used to decompose the PtOEP molecule very efficiently to form metallic nanostructures in the form of 1D nanocolumns and 2D percolated network-layers. The thermal mobilization of the PtOEP molecules at mild temperatures improves the homogeneity and density of the Pt structures, reducing the soft plasma treatment duration. The UV-Vis transmission and XPS analyses probed the total decomposition of the organic counterpart and formation of metallic Pt after the SPE post-deposition treatments.

The transmittance of the fabricated Pt layers and nanocolumns depends strongly on their thickness and the post deposition treatment. We have shown the formation of highly homogeneous semitransparent films with highly flat transmittance spectra in the Visible-Near infrared range and controllable transmission values (from 1 to $70 \%$ transmittance). This behaviour enables their application as highly flat neutral density optical filters.

The deposition conditions of the sacrificial PtOEP layers determine the microstructure of the resulting metallic films. Thus, the transparency and the in-plane conductivity of the Pt nanostructured films have been directly related with the thickness and microstructure of the corresponding films. The poor electrical conductivity results are in line with the SEM analysis since the large gaps observed for the OPVD and RPAVD-Ar and the columnar microstructure in the RPAVD-O2 samples depict a very tortuous path for the current in the sample plane. By contrast, in the case of columnar RPAVD-O2 films the transversal conductivity measurements performed by conducting AFM revealed very low resistance in comparison with commercial ITO thin films. The methodology developed provides a direct route for the implementation of $\mathrm{Pt}$ nanostructured coatings as highly catalytic counter electrodes in a DSC. In fact, the positive results shown also demonstrate the mechanical and chemical stability of the nanoelectrodes processed and operating under real conditions. Concretely, for the low- 
viscosity electrolyte (AN50), a remarkable difference in the photovoltaic behaviour was observed by altering the nanostructure of the deposited platinum counter electrode. It is very interesting to remark that the two samples studied, platinum RPAVD-O2 nanorods (1D) and the RPAVD-Ar networks (2D), outperformed the standard counterelectrode Platisol in terms of the generated photocurrent and photovoltage, although the 2D electrode had a noticeable superior performance. Impedance spectroscopy data showed that the superior catalytic behaviour, supported by the simultaneous enhancement of redox couple regeneration at the counter electrode surface and the subsequent lowering of the recombination rate. In the case of the highviscosity electrolyte, all three counter electrodes behaved quite similarly, except for a higher photocurrent observed in the $1 \mathrm{D}$ one.

The basis of the methodology relays in the use of Pt-porphyrin as vacuum-processable precursor for the formation of Pt percolated networks and nanocolumns. Among the advantages of this methodology we should address: i) Pt-porphyrin is a low toxicity precursor that sublimates at mild temperatures which yields a precursor layers growth rate easily tuned by the experimental conditions. ii) All the procedures applied are vacuum and plasma well-known methods directly expandable to wafer-scale dimensions. iii) A major advantage of the methodology developed in this work is its straightforward application to other commercial available metal- porphyrins and phthalocyanines, opening a promising route for the fabrication of metal, metal oxide and composite nanostructured layers.

\section{Experimental Section}

Fabrication of the nanostructured platinum films: Platinum octaethylporphyrin (PtOEP) was purchased in Frontier Scientific and used as received. The compound was deposited by OPVD and RPAVD on several substrates as n-doped Si (100), fused 
silica, ITO (VisionTek Systems Ltd., $4 \Omega / \square$ ) and FTO (XOP Glass, 12-14 $\Omega / \square$ ) thin films on glass. The substrates were introduced into a vacuum chamber pumped until reaching a base pressure of $2 \times 10^{-5}$ mbar before starting the deposition process. OPVD was carried out at $2 \times 10^{-2}$ mbar of Ar, a nominal growth rate settled in the QCM at $0.35 \AA / s$ and with the substrates at room temperature. Similar conditions were applied for the RPAVD-Ar processes, operating the plasma ECR-MW discharge at $300 \mathrm{~W}$. For the RPAVD depositions the substrates were located between the plasma discharge and the Knudsen sublimation cell facing down the glow discharge at $10 \mathrm{~cm}$ form the plasma zone. ${ }^{24} \mathrm{RPAVD}_{2}$ experiments with a gas mixture of $80 \% \mathrm{O}_{2} / 20 \%$ Ar were carried out at $600 \mathrm{~W}$ and growth rate $0.6 \AA / \mathrm{s}$ in the same geometrical arrangement and conditions of pressure and temperature. The post-treatment of the samples by soft plasma etching was produced in the same reactor and geometrical configuration. Treatment duration, substrate temperature and plasma composition were varied in order to obtain the full mineralization of the PtOEP and reduction of the platinum films. SPE $\left(\mathrm{O}_{2}+\mathrm{Ar}\right)$ label corresponds to etching under $0.02 \mathrm{mbar}\left(20 \% \mathrm{Ar} / 80 \% \mathrm{O}_{2}\right)$ at $400 \mathrm{~W}$ and $\operatorname{SPE}\left(\mathrm{O}_{2}+\mathrm{H}_{2}\right)$ to treatments at $0.02 \mathrm{mbar}\left(50 \% \mathrm{O}_{2} / 50 \% \mathrm{H}_{2}\right)$ at $600 \mathrm{~W}$. In all the cases pure gases were dosed to the plasma reactor through a set of mass flow controllers and mixed within the chamber at reduced pressure. Please note that at such low pressure the mixture $\mathrm{O}_{2}$ and $\mathrm{H}_{2}$ is not explosive. A second post-treatment consisting in annealing under constant gas flow of a mixture 95\% $\mathrm{Ar} / 5 \% \mathrm{H}_{2}$ was carried out in a furnace. The temperature was set to $135^{\circ} \mathrm{C}$ for 2 hours. In order to facilitate the exposition of results the labels of the samples specifically address the main experimental parameters varied during their fabrication.

Characterization. High-resolution SEM images of the samples deposited on silicon wafers were obtained in a Hitachi S4800 microscope, working at different acceleration voltages $(1-5 \mathrm{kV})$. Cross sectional views were obtained by cleaving the $\mathrm{Si}(100)$ 
substrates. XPS characterization and in situ XPS experiments were performed in a Phoibos 100 DLD X-ray spectrometer from SPECS with an attached high vacuum prechamber where all PtOEP depositions and treatments were carried out. The spectra were collected in the pass energy constant mode at a value of $50 \mathrm{eV}$ using a $\mathrm{Mg} \mathrm{K \alpha}$ source. C1s signal at $284.5 \mathrm{eV}$ was utilized for calibration of the binding energy in the spectra. The assignment of the BE to the different elements in the spectra corresponds to the data in reference. ${ }^{31}$ Plasma source used for XPS in situ experiments consisted of a quartz tube where the plasma was excited by means of a resonant cavity connected to a microwave generator. The power was $70 \mathrm{~mW}$ and the oxygen was supplied to the tube up to a pressure of about $2 \times 10^{-1}$ mbar.

Glancing Angle X-ray Diffraction (GAXRD) measurements were carried out in a Panalytical X'PERT PRO diffractometer for an incident angle of $0.2^{\circ}$.

UV-Vis transmission spectra of samples deposited on fused silica slides were recorded in a Cary 100 spectrophotometer in the range from 190 to $900 \mathrm{~nm}$. UV-Vis-NIR transmission spectra were collected in a PerkinElmer Lambda 750 UV/Vis/NIR spectrophotometer.

Electrical characterization was carried out following different procedures. In the first case, the samples were grown on commercial available Pt electrodes separated $100 \mu \mathrm{m}$ (see ESI S10). The cross section is $1.7 \mathrm{~mm}$ long and $300 \mathrm{~nm}$ thickness. The commercial Pt electrodes were previously cleaned in a sonication bath with ethanol and electrically tested showing a resistance in the order or below the detection limit of our apparatus. Additionally, in line four-point probe measurements were also performed to estimate the real sheet resistance of the samples and, in particular cases, in order to overcome the shadowing issues due to the plasma in the deposition of the films on commercial electrodes (see Section S10 and Figure S10). The system consists in four tips separated $3.3 \mathrm{~mm}$. 
AFM characterization gathered on Fig. S4 were taken with a Dulcinea microscope from Nanotec (Madrid, Spain) working in tapping mode and using high frequency $\mathrm{Si}$ cantilevers $(40 \mathrm{~N} / \mathrm{m}, 300 \mathrm{kHz})$. Scanning conductivity measurements in Fig. 5 were carried out with a current sensing module supplied with a conductive tip NT-MDT with a Pt-Ir cover. The diameter of the conductive tip was bigger than $50 \mathrm{~nm}$ and it presented a resonance frequency of $170-310 \mathrm{kHz}$ and a force constant of 5-20 N/m. AFM images were processed with the WSxM free available software from Nanotec. ${ }^{44}$ DSCs fabrication, assembly and characterization. Working electrode. Prior to any deposition, the substrates were rinsed with acetone, isopropanol and absolute ethanol, and heated to 500 ${ }^{\circ} \mathrm{C}$ for 1 hour. $\mathrm{A} \mathrm{TiO}_{2}$ blocking layer was deposited on the $\mathrm{FTO} /$ glass substrates (Xop Glass, $12-14 \Omega / \square)$ by immersion in a TiCl4 $(40 \mathrm{mM})$ solution at $70{ }^{\circ} \mathrm{C}$ for 30 minutes and then dried in air. The active area consisted of $12 \mu \mathrm{m}$ thick films comprising a layer of $8 \mu \mathrm{m}$ of $20 \mathrm{~nm}$ $\mathrm{TiO}_{2}$ nanoparticles (Dyesol@ paste) and a layer of $4 \mu \mathrm{m}$ of $400 \mathrm{~nm} \mathrm{TiO} 2$ particles (scattering layer), both deposited by the screen printing technique on the conducting glass substrates. These electrodes were slowly heated up to $500{ }^{\circ} \mathrm{C}$ (with a plateau of 15 minutes) and then immersed in a solution of $\mathrm{TiCl}_{4}(40 \mathrm{mM})$, heated to $70^{\circ} \mathrm{C}$ for 30 minutes and dried in air. A final heating cycle was performed at $500{ }^{\circ} \mathrm{C}$ during 30 minutes and then allowed to cool down in air. The active area of the cells was $0.16 \mathrm{~cm} 2.35$ The working electrodes were left overnight in a $0.5 \mathrm{mM}$ solution of $\mathrm{N} 719$ dye in ethanol. Afterwards they were rinsed with an ethanol baker and dried in air.

Counter electrodes. A small hole was drilled on them to allow for electrolyte injection at the end of the process. Later, they were cleaned just as the working electrodes. Platisol® Counter electrodes. These electrodes were fabricated distributing $6 \mu \mathrm{L}$ of Platisol® all over the counter electrode area and once dried adding $6 \mu \mathrm{L}$ more. Then, the counter electrodes were put into the furnace at $400{ }^{\circ} \mathrm{C}$ during 5 minutes with a 15 minutes heating ramp. 
Electrolyte solutions. Two different electrolytes were tested. AN50 was purchased from Solaronix and used as received. 100\% Pyr has the following formulation: $0.1 \mathrm{M}$ I2, 0.05M LiI, 0.5M TBP, 0.1M GuSCN, 1M BMII, 1-butyl-1-methylpyrrolidiniumbis (trifluoromethanesulfonyl)imide (Pyr). Pyr was purchased from Solvionic.

Nanostructured electrodes. Two types of nanoelectrodes were tested: "RPAVD-Ar $(420 \mathrm{~nm}) \mathrm{SPE}\left(\mathrm{Ar}+\mathrm{O}_{2}\right) 210^{\circ} \mathrm{C} 240 \mathrm{~min}+$ Anneal $\left(\mathrm{Ar}+\mathrm{H}_{2}\right)$ " and "RPAVD-O $(500 \mathrm{~nm})$ $\operatorname{SPE}\left(\mathrm{Ar}+\mathrm{O}_{2}\right) 210^{\circ} \mathrm{C} 240 \mathrm{~min}+$ Anneal $\left(\mathrm{Ar}+\mathrm{H}_{2}\right) "$.

Sealing of the cells. A frame of a thermoplastic polymer (Surlyn, Solaronix) was placed on the perimeter of the active area and then sandwiched with the counter electrode. The whole cell was heated to $140{ }^{\circ} \mathrm{C}$ under slight pressure to ensure a proper sealing. After that the electrolyte was injected and the hole on the counter electrode sealed with Surlyn and a cover slide glass.

Characterization of DSCs. The solar-cell devices were characterized using a solar simulator with an AM1.5G filter (ABET). A reference solar cell with temperature output (Oriel, 91150) was used for calibration. Electrochemical Impedance Spectroscopy (EIS) measurements were performed under light with perturbations in the $10^{5}-10^{-3} \mathrm{~Hz}$. A LED LUXEON collimated $(540 \mathrm{~nm})$ source and an Autolab/PGSTAT302N potentiostat were used for EIS measurements. Zview equivalent circuit modelling software (Scribner) was used to fit the EIS data, including the distributed element DX11 (transmission line model). Two identical cells per platinum counter electrode configuration were tested.

\section{Supporting Information}

Supporting Information is available from the author.

\section{Acknowledgements}

We thank the Junta de Andalucia (TEP8067, FQM-6900, FQM 1851 and P12-FQM-2265) and the Spanish Ministry of Economy and Competitiveness and Agencia Estatal de Investigación (MAT2013-40852-R, MAT2013-42900-P, MAT2013-47192-C3-3-R, 
MAT2016-79866-R and MINECO-CSIC 201560E055) and the EU through cohesion fund and FEDER programs for financial support. JRS-V and AngelB acknowledge funding from EU project PlasmaPerovSol. This project has received funding from the European Union's Horizon 2020 research and innovation programme under the Marie Skłodowska-Curie grant agreement No 661480.

\section{References}

1 W. Xiong, Y. Zhou, W. Hou, L. Jiang, M. Mahjouri-Samani, J. Park, X. He, Y. Gao, L. Fan, T. Baldacchini, J.-F. Silvain and Y. Lu, Front. Optoelectron. 2015, 8, 351-378.

2 C. Cheng and H. J. Fan, Nano Today 2012, 7, 327-343.

3 K. Ostrikov, E. C. Neyts and M. Meyyappan, Adv. Phys. 2013, 62, 113-224.

4 A. Barranco, A. Borras, A. R. Gonzalez-Elipe and A. Palmero, Prog. Mater. Sci. 2016, $76,59-153$.

5 A. Borras, M. Macias-Montero, P. Romero-Gomez and A. R. Gonzalez-Elipe, J. Phys. Appl. Phys. 2011, 44, 174016.

6 J. Zheng, R. Yang, L. Xie, J. Qu, Y. Liu and X. Li, Adv. Mater. 2010, 22, 1451-1473.

$7 \quad$ P. Brault, Plasma Process. Polym. 2016, 13, 10-18.

8 P. Brault, Surf. Coat. Technol. 2011, 205, Supplement 2, S15-S23.

9 A. Chen and P. Holt-Hindle, Chem. Rev. 2010, 110, 3767-3804.

10 Y. Ding and M. Chen, MRS Bull. 2009, 34, 569-576.

11 L. Hu, H. Wu and Y. Cui, MRS Bull. 2011, 36, 760-765.

12 D. Angmo and F. C. Krebs, J. Appl. Polym. Sci. 2013, 129, 1-14.

13 S. Thomas, T. G. Deepak, G. S. Anjusree, T. A. Arun, S. V. Nair and A. S. Nair, J. Mater. Chem. A 2014, 2, 4474-4490.

14 L. Hu, J. W. Choi, Y. Yang, S. Jeong, F. L. Mantia, L.-F. Cui and Y. Cui, Proc. Natl. Acad. Sci. 2009, 106, 21490-21494. 
15 D. Chen, Y.-E. Miao and T. Liu, ACS Appl. Mater. Interfaces 2013, 5, 1206-1212.

16 D. Langley, G. Giusti, C. Mayousse, C. Celle, D. Bellet and J.-P. Simonato, Nanotechnology 2013, 24, 452001.

17 J. Kim, J. Kang, U. Jeong, H. Kim and H. Lee, ACS Appl. Mater. Interfaces 2013, 5, $3176-3181$.

18 H. Y. Jang, S.-K. Lee, S. H. Cho, J.-H. Ahn and S. Park, Chem. Mater. 2013, 25, $3535-3538$.

19 A. Borras, O. Gröning, M. Aguirre, F. Gramm and P. Gröning, Langmuir 2010, 26, $5763-5771$.

20 A. Borras, M. Aguirre, O. Groening, C. Lopez-Cartes and P. Groening, Chem. Mater. 2008, 20, 7371-7373.

21 A. L. Briseno, S. C. B. Mannsfeld, S. A. Jenekhe, Z. Bao and Y. Xia, Mater. Today 2008, $11,38-47$.

22 F. J. Aparicio, M. Holgado, A. Borras, I. Blaszczyk-Lezak, A. Griol, C. A. Barrios, R. Casquel, F. J. Sanza, H. Sohlström, M. Antelius, A. R. González-Elipe and A. Barranco, Adv. Mater. 2011, 23, 761-765.

23 F. J. Aparicio, I. Blaszczyk-Lezak, J. R. Sánchez-Valencia, M. Alcaire, J. C. González, C. Serra, A. R. González-Elipe and A. Barranco, J. Phys. Chem. C 2012, 116, 8731-8740. 24 F. J. Aparicio, M. Alcaire, A. Borras, J. C. Gonzalez, F. López-Arbeloa, I. BlaszczykLezak, A. R. González-Elipe and A. Barranco, J. Mater. Chem. C 2014, 2, 6561-6573. 25 M. Macias-Montero, A. N. Filippin, Z. Saghi, F. J. Aparicio, A. Barranco, J. P. Espinos, F. Frutos, A. R. Gonzalez-Elipe and A. Borras, Adv. Funct. Mater. 2013, 23, 59815989.

26 F. J. Aparicio, G. Lozano, I. Blaszczyk-Lezak, Á. Barranco and H. Míguez, Chem. Mater. 2010, 22, 379-385. 
M. Alcaire, J. R. Sanchez-Valencia, F. J. Aparicio, Z. Saghi, J. C. Gonzalez-Gonzalez,

A. Barranco, Y. O. Zian, A. R. Gonzalez-Elipe, P. Midgley, J. P. Espinos, P. Groening and A. Borras, Nanoscale 2011, 3, 4554.

28 M. Alcaire, A. N. Filippin, M. Macias-Montero, J. R. Sanchez-Valencia, T. C. Rojas, A. Mora-Boza, C. Lopez-Santos, J. P. Espinos, A. Barranco and A. Borras, Plasma Process. Polym. 2016, 13, 287-297.

29 I. Blaszczyk-Lezak, F. J. Aparicio, A. Borras, A. Barranco, A. Álvarez-Herrero, M. Fernández-Rodríguez and A. R. González-Elipe, J. Phys. Chem. C 2009, 113, 431-438

30 I. N. Leontyev, A. B. Kuriganova, N. G. Leontyev, L. Hennet, A. Rakhmatullin, N. V. Smirnova and V. Dmitriev, RSC Adv. 2014, 4, 35959-35965.

31 A. V. Naumkin, A. Kraut-Vass, S. W. Gaarenstroom and C. J. Powell. NIST X-Ray Photoelectron Spec-troscopy Database, Version 4.1 (National Institute of Standards and Technology, Gaithersburg, 2012).

32 A. Borras, A. Yanguas-Gil, A. Barranco, J. Cotrino and A. R. Gonzalez-Elipe, Phys. Rev. B 2007, 76, 235303.

33 A. Borras, J. Cotrino and A. R. Gonzalez-Elipe, J. Electrochem. Soc. 2007, 154, P152P157.

34 A. Borras, J. R. Sanchez-Valencia, J. Garrido-Molinero, A. Barranco and A. R. González-Elipe, Micro. Meso. Mater. 2009, 118, 314-324.

35 A. Fridman, Organic and Polymer Plasma Chemistry on Plasma Chemistry, Chapter 9, pp 589-675, Cambridge University Press, Cambridge, 2008.

36 F. S. Denes and S. Manolache, Prog. Polym. Sci. 2004, 29, 8.

37 A. K. Bansal, W. Holzer, A. Penzkofer and T. Tsuboi, Chem. Phys. 2006, 330, 118129. 
38 M. M. Da Silva, A. R. Vaz, S. A. Moshkalev and J. W. Swart, ECS 2007, 9, pp. 235241.

39 W. Kubo, S. Kambe, S. Nakade, T. Kitamura, K. Hanabusa, Y. Wada and S. Yanagida, J. Phys. Chem. B 2003, 107, 4374-4381.

40 P. Kuang, J.-M. Park, W. Leung, R. C. Mahadevapuram, K. S. Nalwa, T.-G. Kim, S. Chaudhary, K.-M. Ho and K. Constant, Adv. Mater. 2011, 23, 2469-2473.

41 J. Idigoras, R. Tena-Zaera and J. A. Anta, Phys. Chem. Chem. Phys. 2014, 16, 2151321523.

42 Q. Wang, S. Ito, M. Grätzel, F. Fabregat-Santiago, I. Mora-Seró, J. Bisquert, T.

Bessho and H. Imai, J. Phys. Chem. B, 2006, 110, 25210-25221.

43 J. Bisquert, F. Fabregat-Santiago, I. Mora-Seró, G. Garcia-Belmonte and S. Giménez, J. Phys. Chem. C, 2009, 113, 17278-17290.

44 I. Horcas, R. Fernández, J. M. Gómez-Rodríguez, J. Colchero, J. Gómez-Herrero and A. M. Baro, Rev. Sci. Instrum. 2007, 78, 13705. 